\title{
Terahertz induced optical second harmonic generation from silicon surface
}

\author{
S.B. Bodrov ${ }^{1,2}$, A.I. Korytin ${ }^{1}$, Yu.A. Sergeev ${ }^{1}$, A.N. Stepanov ${ }^{1}$ \\ ${ }^{1}$ Institute of Applied Physics of the Russian Academy of Sciences, Nizhny Novgorod, Russia, yas@ufp.appl.sci-nnov.ru \\ ${ }^{2}$ University of Nizhny Novgorod, Nizhny Novgorod, Russia
}

Second harmonic generation (SHG) of optical radiation from surface is one effective technique for surface diagnostics [1]. It is well known, that in bulk media with inversion center the second harmonic generation is prohibited in the dipole approximation. However, the surface breaks the symmetry and allows SHG. In this regard, the second harmonic $(\mathrm{SH})$ signal is extremely sensitive to the state of the surface. The SHG technique allows to measure inhomogeneities of the surface, dynamics of fast processes, such as fast melting under the action of femtosecond laser pulses, contains information about the surface states of the material. An application of the external field on the surface can significantly change the second harmonic signal $[2,3]$.

In this work, we investigated influence of intense THz field on SHG from the silicon surface (111).

A Ti:Sp femtosecond laser system at $795 \mathrm{~nm}$ with energy of $0.7 \mathrm{~mJ}$, duration of $70 \mathrm{fs}$ and repetition rate of $700 \mathrm{~Hz}$ was used in the experiment. Optical radiation was divided into two beams (see Fig.1). The pump beam was used to generate $\mathrm{THz}$ radiation by the tilted intensity front technique in a $\mathrm{LiNbO} 3$ crystal

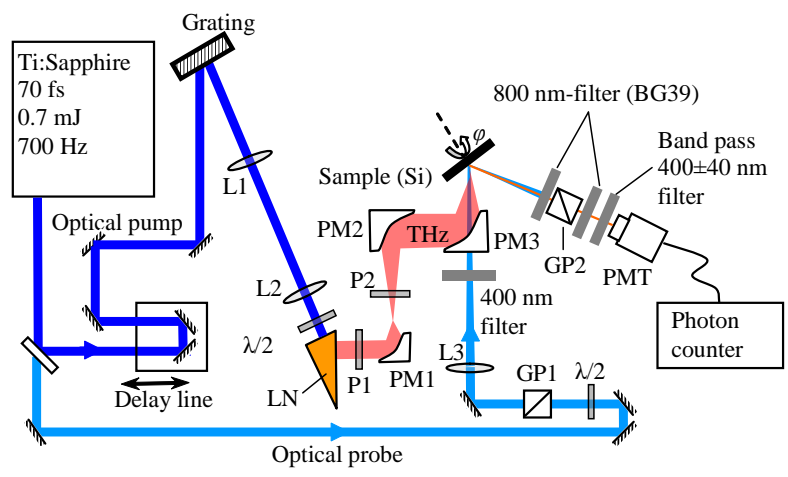

[4,5]. The terahertz radiation was

Fig. 1. Experimental setup (L1, L2, L3- lenses; PM1, PM2, PM3 - parabolic mirrors; LN - LiNbO3; GP1, GP2 - GlanTaylor prism; $\mathrm{P} 1, \mathrm{P} 2-\mathrm{THz}$ polarizer)

focused on the sample using a system of off-axis parabolas. The maximum $\mathrm{THz}$ field in focus reached a value of $300 \mathrm{kV} / \mathrm{cm}$. For the attenuation of THz fields were used two $\mathrm{THz}$ polarizer. A probe laser pulse focused on the sample in the spot of about $100 \mu \mathrm{m}$ (smaller than the size of the THz spot). The second harmonic radiation generated from the surface was detected by the PMT connected to the pulse counting system. The polarization properties of the SH was controlled by polarizer GP2. The special filters was used to block laser radiation.

In the course of the experiment, the generation of second harmonic at different combinations of polari- zation of optical and $\mathrm{THz}$ radiation was investigated. Under application of $\mathrm{THz}$ field, the $\mathrm{SH}$ signal was significantly increased when second harmonic and $\mathrm{THz}$ electric fields were parallel. In the case when $\mathrm{THz}$, first and second harmonic electric fields have $s$ polarization, the $\mathrm{SH}$ signal increased by 30 times. The dependences of the SH signal on the energy of optical and $\mathrm{THz}$ pulses, on the delay time between them, and the dependence on the azimuthal angle (rotation angle of the sample) were investigated. In Fig. 2 an example of angular dependence is presented, where all fields (THz field, field of the first and second harmonics) have $p$ polarization.

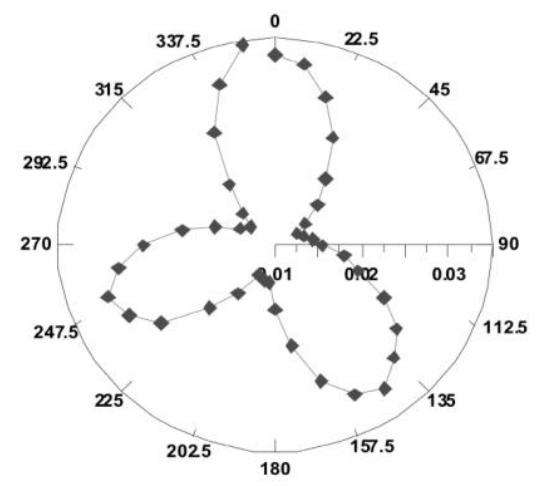

Fig. 2. Angular dependence of SH intensity: optics Ppolarization, THz field P-polarization, SH P-polarization

To describe the experimental results, the phenomenological theory of second harmonic generation from (111) silicon surface was developed with account of presents of $\mathrm{THz}$ field. By comparison with experimental data, it was found that $\mathrm{THz}$ induced source of $\mathrm{SH}$ is in phase with the source of $\mathrm{SH}$ without $\mathrm{THz}$ field.

\section{References}

1. Bloembergen, N., Chang, R. K., Jha, S. S., Lee, C. $H$. Second-harmonic generation of light in reflection from media with inversion symmetry // Phys. Rev. 1966. V. 16, No. 22. P. 986-989.

2. Nahata, A., Heinz, T.F. Detection of freely propagating terahertz radiation by use of optical second-harmonic generation // Opt. Letters, 1998. V. 23, No. 1. P. 67-69.

3. Lee, C. H., Chang, R. K., Bloembergen N. Nonlinear electroreflection in silicon and silver// Phys. Rev. Lett. 1967. V. 18, No 5. P. 167-170.

4. Fulop, J. A., Palfalvi, L., Almasi, G., Heblin, J. Design of high-energy terahertz sources based on optical rectification // Opt. Express, 2010. V. 18, No. 12. P. 1231112327

5. Bodrov, S. B., Murzanev, A. A., Sergeev, Y.A., Malkov, Y.A., Stepanov A.N. Terahertz generation by tiltedfront laser pulses in weakly and strongly nonlinear regimes // Applied Physics Letters. 2013. V.103, No. 25. P. 251103 